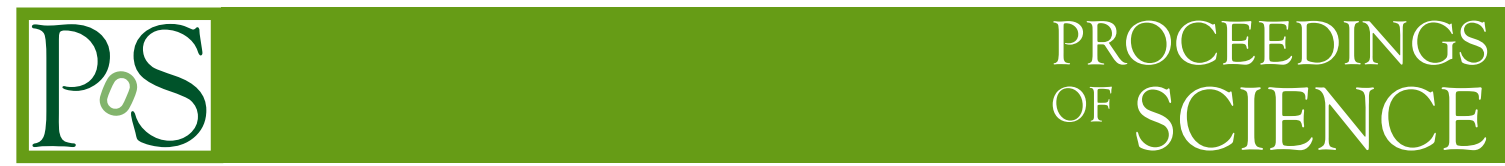

\title{
Recent results from RHIC
}

\section{Alexander Schmah* Lawcrence Berkeley National Laboratory}

E-mail: aschmah@lbl.gov

The first part of the beam energy scan (BES) program at RHIC was successfully completed in the years 2010 and 2011. Furthermore high statistics data at $\sqrt{s_{N N}}=200 \mathrm{GeV}$ was taken in the recent years at STAR and PHENIX. First results from BES data show that particle yield measurements are in good agreement with previously published data from SPS and AGS experiments. In addition, new observations like the centrality and energy dependence of chemical freeze-out parameters $\left(T_{\mathrm{ch}}\right.$ and $\left.\mu_{B}\right), \mathrm{R}_{C P}$, di-lepton spectra, or the smoothly increasing difference with decreasing energy in the elliptic flow $v_{2}$ between particles and corresponding anti-particles, are discussed.

International Winter Meeting on Nuclear Physics,

21-25 January 2013

Bormio, Italy

\footnotetext{
* Speaker.
} 

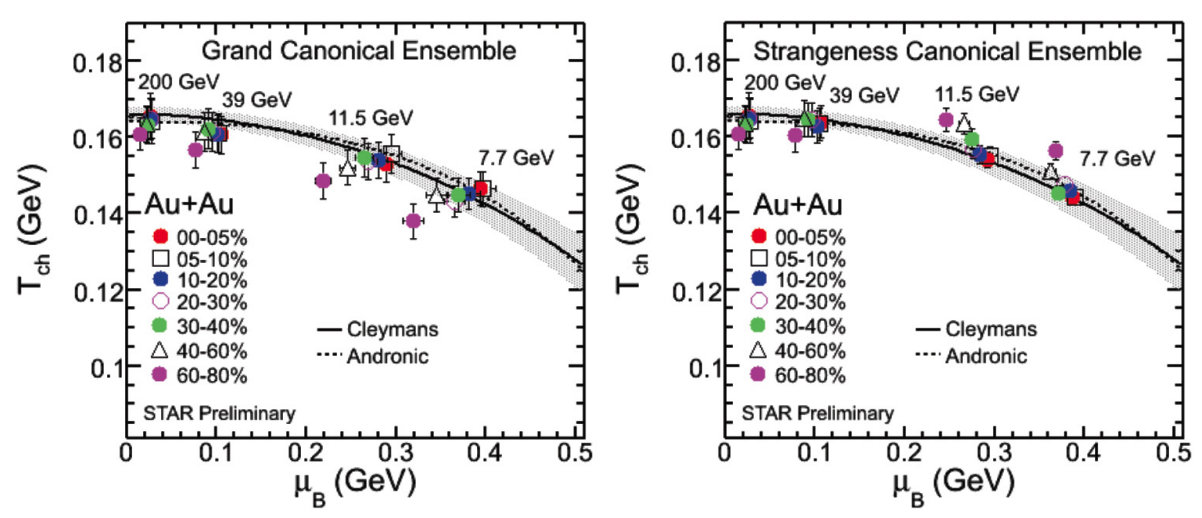

Figure 1: (Color online) Centrality dependence of the chemical freeze-out parameters $T_{c h}$ and $\mu_{B}$ for $\sqrt{s_{N N}}$ $=7.7,11.5,39$ and $200 \mathrm{GeV}$ for the grand canonical fit (left) and the strangeness canonical fit (right).

\section{Introduction}

Several heavy-ion measurements from the past decade indicate the existence of a QCD phase with partonic degrees of freedom at high energy A+A collisions. The quark-gluon plasma (QGP) is expected to have fundamentally different characteristics compared to the hadron gas phase which is observed at lower energies. Lattice QCD calculations show a crossover between the hadron gas and the QGP phase at low baryon chemical potentials $\mu_{B}$ [1], whereas a first order phase transition is expected at higher $\mu_{B}$ values. This would result in a QCD critical point. The two main goals of the beam energy scan (BES) at the Relativistic Heavy-Ion Collider (RHIC) facility are to find direct signatures for a transition between the two phases and for the critical point [2]. For this purpose, a series of $\mathrm{Au}+\mathrm{Au}$ experiments at different energies were accomplished in the years 2010 and 2011 at RHIC. In addition high statistics data for the collision system $\mathrm{Au}+\mathrm{Au}$ at $\sqrt{s_{N N}}=200 \mathrm{GeV}$ was recorded at STAR and PHENIX. This is needed for rare probes like lepton pairs from vector meson decays. Latter ones are due to their small interaction cross section with hadronic matter penetrating probes and used to study the whole evolution of the created matter.

Within the range of the chosen BES energies it is expected that fireballs are created which exhibit properties around this transition line. For each of these energies multiple observables like azimuthal anisotropies $\left(v_{n}\right), p_{T}$ spectra, particle yields and ratios, azimuthal HBT, di-lepton spectra, $R_{\mathrm{CP}}$ etc. are studied. A rapid change at a certain energy of one or more of these observables could indicate an underlying phase transition. In addition, the disappearance of QGP signatures like number-of-constituent-quark (NCQ) scaling are studied as a function of energy. Comparisons to model predictions are performed as well, to identify an agreement or significant differences with the data. To find signatures of the critical point event-by-event measurements are performed such as the net proton or $K / \pi$ fluctuation measurements.

\section{Results}

In the following sections, a selection of $\sqrt{s_{N N}}=200 \mathrm{GeV}$ and BES results are highlighted. The first part shows the energy dependence of the chemical freeze-out conditions. In the next two sections di-lepton spectra and the charged hadron nuclear modification factor $R_{\mathrm{CP}}$ are discussed in 
comparison with model calculations. It is followed by recent results from identified particle elliptic flow and higher moments from net-proton distributions.

\subsection{Chemical freeze-out conditions}

Bulk properties from heavy-ion reactions, such as the chemical and kinetic freeze-out temperatures, can be extracted from particle spectra. Figure 1 shows the chemical freeze-out parameters $T_{\mathrm{ch}}$ and $\mu_{B}$ for various energies and centralities [3], extracted from THERMUS [4] fits to particle ratio yields from a grand canonical ensemble fit (left) and a strangeness canonical ensemble fit (right). A centrality dependence of these parameters is observed at the lower energies 39, 11.5 and 7.7 GeV, whereas the parameters seem to be constant at $200 \mathrm{GeV}$. The temperature dependence between the two ensembles is slightly different. At SIS energies a similar study was performed [5].

\subsection{Di-electron spectra}

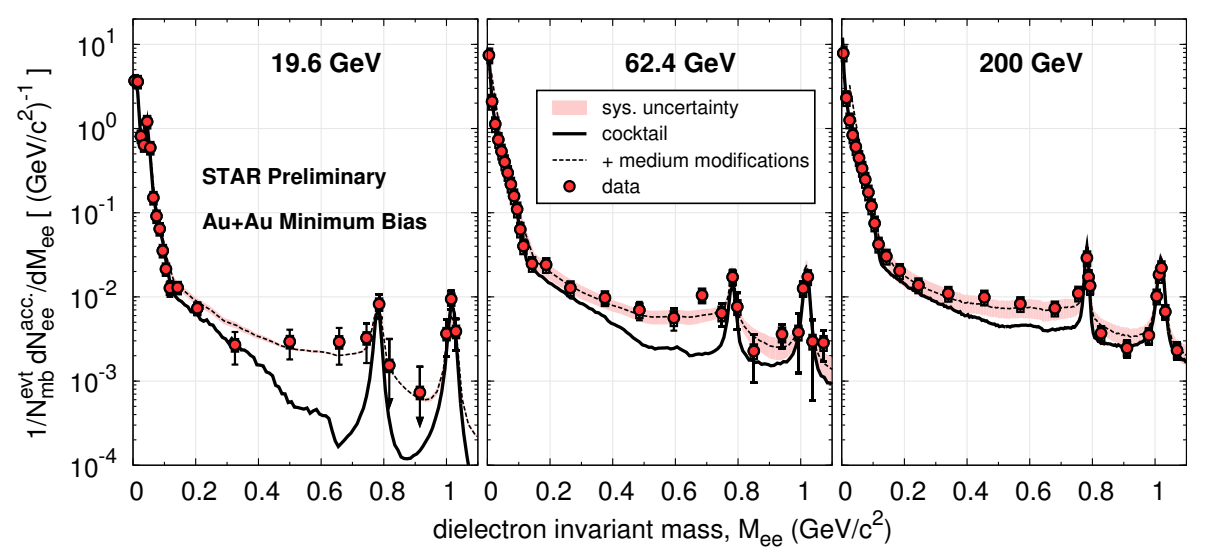

Figure 2: (Color online) Di-electron invariant mass distributions from $\sqrt{s_{N N}}=19.6,62.4$, and $200 \mathrm{GeV} 0-$ $80 \%$ central $\mathrm{Au}+\mathrm{Au}$ collisions. The data points contain statistical and systematic error bars. The solid black line shows a cocktail simulation without the $\rho^{0}$ contributions. The dashed lined is the sum of the cocktail simulation and a model calculation with an in-medium modified $\rho^{0}$.

In Fig. 2 the di-electron invariant mass distributions from $\sqrt{s_{N N}}=19.6,62.4$, and $200 \mathrm{GeV}$ 0-80\% central $\mathrm{Au}+\mathrm{Au}$ collisions are shown in comparison with cocktail and model calculations [6]. The combinatorial and correlated background was subtracted from the data and it is corrected for efficiency. Conversion pairs were removed at $\sqrt{s_{N N}}=62.4$ and $200 \mathrm{GeV}$ but not at $19.6 \mathrm{GeV}$. In the vacuum cocktail simulation the $\rho^{0}$ contribution was not included since this vector meson has a short live time and decays inside the dense medium which is expected to modify the spectral function. This modification is included in the sum of the cocktail and model calculations [7, 8] shown as dashed lines. In this model a complete evolution of the QGP and thermal dilepton rates in the QGP and hadron-gas (HG) phases are convoluted with an isentropic fireball evolution. The model plus cocktail calculations can describe the low mass part of the di-electron spectra over a wide range of energies. 

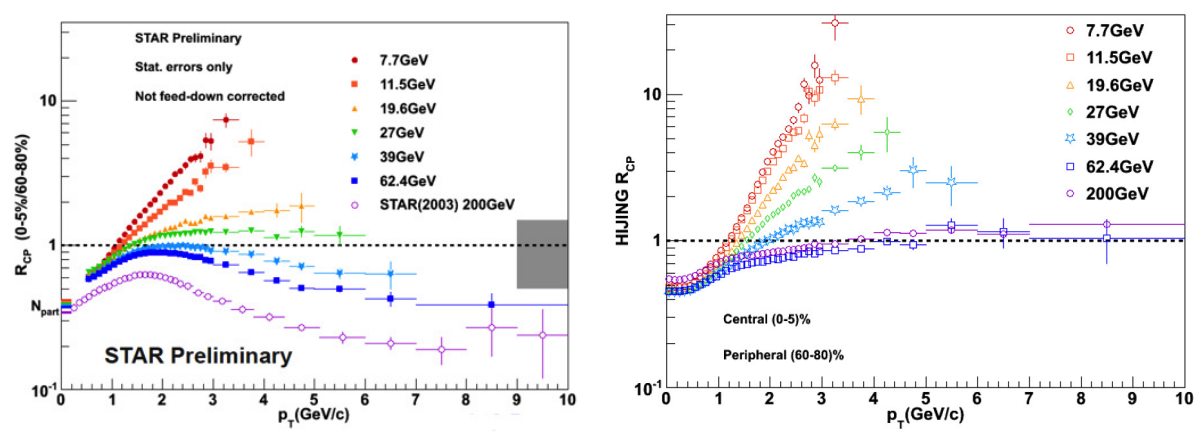

Figure 3: (Color online) The nuclear modification factor $R_{\mathrm{CP}}$ for unidentified charged hadrons from different energies of the BES (left) and from HIJING calculations (right).

\subsection{Nuclear modification factor $R_{\mathbf{C P}}$}

The energy loss of high energetic partons in heavy-ion reactions and the corresponding suppression in the yield is one of the key signatures for the QGP phase [9]. Figure 3 shows the nuclear modification factor $R_{\mathrm{CP}}$ of unidentified charged hadrons for various energies from the BES (left) and for HIJING calculations (right). The data is corrected for detector efficiency. The data shows a clear suppression at high transverse momenta at $\sqrt{s_{N N}}=39,62.4$, and $200 \mathrm{GeV}$, whereas the $R_{\mathrm{CP}}$ becomes larger than 1 at lower energies. The HIJING simulations include the Cronin effect through $\mathrm{k}_{\mathrm{T}}$ broadening but have in-medium energy loss turned off. The $R_{\mathrm{CP}}$ flattens out to 1 at high $p_{\mathrm{T}}$ for $\sqrt{s_{N N}}=62.4$ and $200 \mathrm{GeV}$, as we would expect for jets that do not experience in-medium effects, but at lower collision energies we see a significant enhancement that is qualitatively very similar to what we observe in data. This suggests that for the lower collision energies the Cronin effect becomes more dominant, which makes it more difficult to draw conclusions about the magnitude of partonic energy loss.

\subsection{Elliptic flow of identified particles}

The elliptic flow $v_{2}$ is the second order harmonic of the azimuthal particle distribution relative to the reaction plane. It is generated by the pressure gradient of the overlapping nuclei, mainly during the early stage of the reaction. The number-of-constituent quark (NCQ) scaling of $v_{2}$ at top RHIC energies is interpreted as one of the signatures for the QGP phase [10]. On the left hand side of Fig. 4 the energy dependence of the mean difference in $v_{2}$ between particles and their corresponding anti-particles is plotted. At higher energies we observe a negligible difference between $K^{+}$and $K^{-}$, and between $\pi^{+}$and $\pi^{-}$. A difference of about 0.005 is found for baryons at 39 and $62.4 \mathrm{GeV}$. This constant behaviour changes at energies below $27 \mathrm{GeV}$. Baryons show an increase with a magnitude in the $v_{2}$ difference of up to 0.05 . Pions show the opposite behaviour compared to kaons with respect to their charge. On the right hand side of Fig. 4 the dependence of the $v_{2}$ difference as a function of the baryon chemical potential is shown. The values of $\mu_{B}$ are from the parametrization of Ref. [11]. A linear increase of the difference with $\mu_{B}$ is observed which could point to a direct connection between the two quantities. From the observed difference in $v_{2}$ between particles and corresponding anti-particles at lower energies, which also holds for 

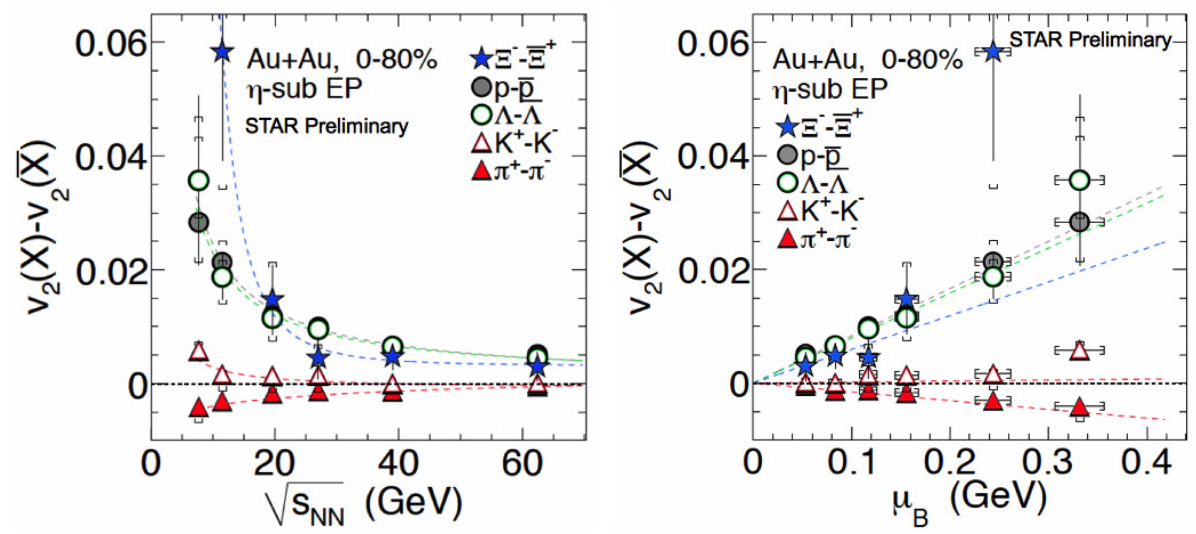

Figure 4: (Color online) The difference in $v_{2}$ between particles $(\mathrm{X})$ and their corresponding anti-particles $(\overline{\mathrm{X}})$ (see legend) as a function of $\sqrt{s_{N N}}$ (left) and the baryon chemical potential $\mu_{B}$ (right) for $0-80 \%$ central $\mathrm{Au}+\mathrm{Au}$ reactions.

the transverse momentum dependence, one can infer that the NCQ scaling between these particles is broken. The splitting could be an indication that hadronic interactions become more important at lower energies. Also other effects like transported quarks or hadronic potentials could explain qualitatively the observations as shown in $[12,13]$.

\subsection{Higher moments of net-proton distributions}

The existence of a QCD critical point would most probably lead to a significant increase of event-by-event fluctuations for $T-\mu_{B}$ trajectories which come close to the critical area or pass the phase transition. It was suggested to study observables which have a connection to baryon number conservation like net-proton distributions [14]. In Fig. 5 the net-proton moment products $\kappa \sigma^{2}$ and $S \sigma$, which are connected to baryon number susceptibilities and the correlation length, are shown in comparison with Poisson and UrQMD calculations for four different centrality bins [15]. The $\kappa \sigma^{2}$ and normalized $S \sigma$ for the most central 0-5\% Au+Au reactions are for all energies below the Poisson expectation, whereas the results from more peripheral reactions show values above unity at the lowest energies and below unity for higher energies.

\section{Summary and outlook}

The first results from the beam energy scan program at RHIC contain important new systematics for the search of the QCD phase transition and the critical point. Event-by-event dynamical fluctuations, particle spectra and bulk correlations like elliptic and directed flow were studied as a function of energy and centrality. A centrality dependence of the chemical freeze-out parameters was observed which shows a slight difference between the grand canonical and strangeness canonical calculation. A comparison of low mass di-leptons pairs to model calculations show a good agreement for $\sqrt{s_{N N}}=19.6,62.4$, and $200 \mathrm{GeV}$. Further energies will be studied by STAR and PHENIX in the future. The nuclear modification factor $R_{\mathrm{CP}}$ shows a suppression at high $\mathrm{p}_{T}$ for energies above $\sqrt{s_{N N}}=27 \mathrm{GeV}$ and values above unity for the lower energies. HIJING model calculations show a similar trend. For the first time an energy dependence of particle-antiparticle 


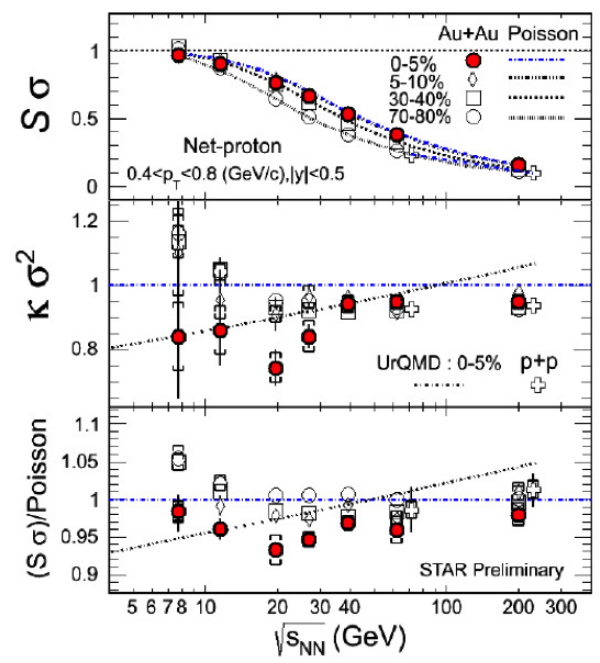

Figure 5: (Color online) Energy dependence of $\kappa \sigma^{2}$ and $S \sigma$ of net-proton distributions for different centralities of $\mathrm{Au}+\mathrm{Au}$ reactions. The STAR data is compared with Poisson expectations and UrQMD transport model calculations.

$v_{2}$ difference was measured from which one can infer that the number-of-constituent quark scaling between particles and anti-particles is broken at the lower energies. The net-proton higher moment products $\kappa \sigma^{2}$ and $S \sigma$ for most central $\mathrm{Au}+\mathrm{Au}$ collisions are below the Poisson expectation whereas relative values above unity are observed for lower energies and more peripheral collisions. It is planned to have another phase of BES experiments at RHIC, starting from the year 2015.

\section{References}

[1] Y. Aoki, G. Endrodi, Z. Fodor, S. D. Katz, K. K. Szabo, Nature, 443 (2006) 675

[2] M. M. Aggarwal et al. (STAR Collaboration), arXiv:1007.2613 [nucl-ex] (2010)

[3] S. Das (STAR Collaboration), arXiv:1210.6099 [nucl-ex], (2012)

[4] S. Wheaton, J. Cleymans, M. Hauer, Computer Physics Communications, 180 (2009)

[5] J. Cleymans, H. Oeschler and K. Redlich, Phys. Rev. C 59, 1663 (1999)

[6] F. Geurts (STAR Collaboration), arXiv:1210.5549 [nucl-ex], (2012)

[7] R. Rapp, J. Wambach, H. van Hees, arXiv:0901.3289v1 [hep-ph]

[8] R. Rapp, Phys. Rev. C 63, 054907; R. Rapp, private communication (2001)

[9] J. Adams et al. (STAR Collaboration), Phys. Rev. Lett. 91172302 (2003)

[10] J. Adams et al. (STAR Collaboration), Phys. Rev. Lett. 92, 052302 (2004)

[11] S. K. Tiwari, P. K. Srivastava and C. P. Singh, Phys. Rev. C 85, 014908 (2012)

[12] J. C. Dunlop, M. A. Lisa, and P. Sorensen, Phys. Rev. C 84, 044914 (2011)

[13] J. Xu, L. -W. Chen, C. M. Ko, and Z. -W. Lin, Phys. Rev. C 85, 041901 (2012)

[14] V. Koch, A. Majumder and J. Randrup, Phys. Rev. Lett. 95, 182301 (2005)

[15] X. Luo (STAR Collaboration), arXiv:1210.5573 [nucl-ex], (2012) 